\title{
Lupus Vulgaris of the External Nose in a Paediatric Patient: A Case Report from Muhimbili National
} Hospital, Tanzania

Abraham Zephania Saitabau ${ }^{1 *}$, Moshi Ndeserua², Richard Enica³ ${ }^{3}$ Daudi Ntunaguzi ${ }^{4}$

${ }^{1}$ Department of Surgery, University of Dodoma, College of Health Sciences, Tanzania

${ }^{2}$ Department of Otorbinolaryngology, Mubimbili University of Health and Allied Sciences, Tanzania

${ }^{3}$ Department of Otorbinolaryngology, Mubimbili University of Health and Allied Sciences, Tanzania

${ }^{4}$ Department of Otorbinolaryngology, Mubimbili University of Health and Allied Sciences, Tanzania

*Corresponding Author: Abraham Zephania Saitabau, email: zsaitabau@yahoo.com

\begin{abstract}
Lupus vulgaris is the most common form of cutaneous tuberculosis which usually occurs in patients who have been previously sensitized to Mycobacterium tuberculosis. [1] We present a case of a 4-year-old girl who was diagnosed to have lupus vulgaris clinically and was then confirmed histopathologically. On local examination, the patient had irregularly bordered, well demarcated, whitish to reddish lesion on her external nose. The histopathological examination showed many dermal stromal granulomas of epithelioid cells, many multinucleated giant cells of Langhans type. This case report is therefore to emphasize on the importance of early diagnosis of lupus vulgaris of the external nose both clinically and on histopathological basis so as to avoid its destructive consequences which are mainly erosion of the external nose, nasal cavity and the face and in rare occasions, possible development of a carcinoma of squamous cell type.
\end{abstract}

Key words: Lupus vulgaris, external nose, Tanzania

\section{Introduction}

By definition, lupus vulgaris is a progressive form of cutaneous tuberculosis which occurs in patients who have been previously sensitized to Mycobacterium tuberculosis and the disease is acquired either endogenously by haematogenous or exogenously by direct inoculation of the bacilli into the skin or lymphatic spread from an underlying infected focus in a sensitized host with a moderate to high degree of immunity to Mycobacterium tuberculosis.[1] It's progressive in nature if no any medical intervention is undertaken to control the disease.

In developing countries like Tanzania, tuberculosis has kept on drawing attention from healthcare professionals and the society in general because of its multi-systemic effect, the increasing incidence of multiple drug resistant $\mathrm{TB}$ and the surge of HIV/TB co-infection which is of concern in many developing countries including Tanzania.[2]

Early diagnosis of lupus vulgaris is of paramount importance if its associated consequences including mid-facial destruction and possible development of carcinoma of the external nose are to be avoided and this is through high index of suspicion by all clinicians at all levels of healthcare provision including primary healthcare facilities. Establishment of its differential diagnoses is not an easy task and it should be based on clinical grounds, histopathological or microbiological examinations and Otorhinolaryngologists should be involved whenever a patient has an ulcer on the external nose that does not respond to usual antibiotics given in a local setting.[3]

We are reporting a 4-year-old girl who was diagnosed to have lupus vulgaris of the external nose with emphasis on early clinico-histopathological diagnosis of the disease so that early treatment is instituted to avoid its destructive consequences which causes mid-facial erosion and possible development of a squamous cell carcinoma of the external nose in rare occasions. To the best of our knowledge, this is a first case report of lupus vulgaris of the external nose in Tanzania.

\section{Case Report}

The 4-year old girl presented with a three month history of a swelling on the external nose with no history of nasal bleeding, nasal obstruction, or constitutional symptoms of tuberculosis. The patient had a positive history of open tuberculosis contact with her grandmother who had pulmonary tuberculosis about a year prior to the presentation of the current complaints by the patient and it was reported that the grandmother was kept on a 6-months course of Anti-tuberculous chemotherapy and was cured. On local examination the patient had well demarcated, irregularly bordered, whitish to reddish lesion on her external nose. The patient had neither palpable peripheral lymph nodes nor systemic 
examination abnormalities. However, the patient has received all immunizations as per the immunization and vaccine development programme of Tanzania.

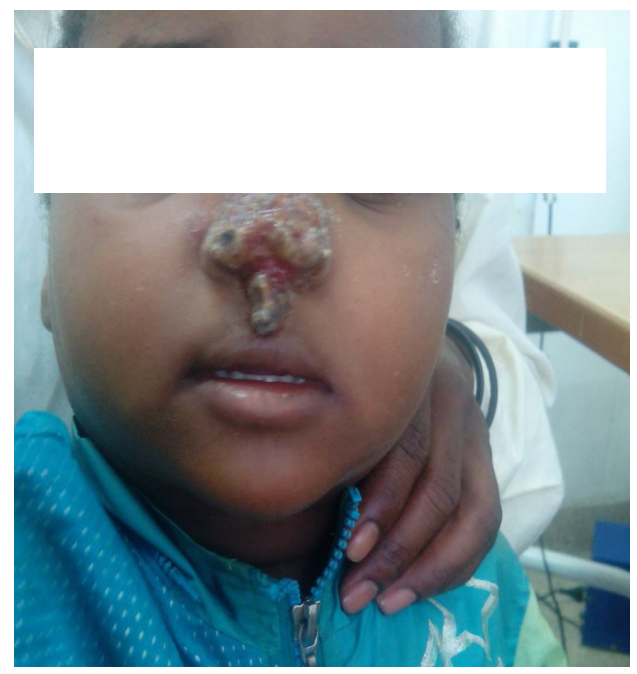

Figure 1 A 4-year old child with lupus vulgaris of the external nose diagnosed both clinically and confirmed on histopathological basis upon taking a wedge biopsy.

To investigate the patient, the following were the results; Complete blood count and Urinalysis which were normal. Erythrocyte sedimentation rate was $18 \mathrm{~mm} /$ hour and HIV screening was negative. The patient had no signs or symptoms of pulmonary tuberculosis and Chest radiograph was normal. The patient was referred to Tuberculosis Unit and is on Anti-tuberculous drugs (Isoniazid, Rifampicin, Pyrazinamide and Ethambutol) for two weeks now.

\section{Discussion}

It was once known for cutaneous tuberculosis to be a common disease but has now become rare because of improved living conditions, BCG vaccination and effective antituberculous drugs. If no intervention is undertaken then the course of lupus vulgaris becomes extremely chronic and thus extending over many years and its ultimate consequences become unavoidable.[4] There are reported periods of relative inactivity in lupus vulgaris but generally it leads to considerable impairment of function and disfiguration because of its progressive nature.[5] The most serious complication of untreated lupus vulgaris is the development of carcinoma of the external nose.[1,6]

With particular emphasis in children, most cases of primary tuberculosis are contracted on the extremities and on the face following scratches, bruising, lacerations, pin-pricks, impetigo, boils piercing, tattoos, and circumcision. $[1,4]$

Malignant transformation of lupus vulgaris to lupus carcinoma has been reported in certain parts of the World and it's estimated to range from $0.5 \%$ to
$10.5 \%$. The interval from its onset to the occurrence of malignancy ranges from 2 to 79 years.[4] Following exogenous inoculation, lupus vulgaris may manifest at the site of BCG vaccination and may also be acquired endogenously through reactivation of dormant or persistent Mycobacterium tuberculosis after a reduction of cell-mediated immunity.[1,2,7]

Establishing the diagnosis of lupus vulgaris is not a straightforward task and clinicians and Otorhinolaryngologists need to have a high index of suspicion. The diagnosis should mainly be based on clinico-histopathological findings and retrospective review of response to treatment;[1] relying on tissue culture and polymerase chain reaction often is of no help because of low yield.[8] The diagnosis of the child in this case report was established on clinicohistopathological basis following a wedge biopsy.

\section{Conclusion}

The aim of this case report is to emphasize on the role of early diagnosis by having a high index of suspicion of any form of cutaneous tuberculosis like lupus vulgaris of the external nose so that early initiation of antituberculous drugs is done so as to avoid its looming consequences like disfiguration of the external nose and nasal cavity, and rarely development of squamous cell carcinoma of the external nose.

\section{References}

1. Afsar FS, Afsar I, Diniz G, Asilsoy S, Sorguc Y. Lupus vulgaris in a pediatric patient: a clinicohistopathological diagnosis. Brazilian J Infect Dis [Internet]. 2008;12:152-4.

2. Dias MFRG, Bernardes Filho F, Quaresma MV, do Nascimento LV, Nery JA da C, Azulay DR. Update on cutaneous tuberculosis. An Bras Dermatol. 2014;89:925-38.

3. Cheng VCC, Yam WC, Hung IFN, Woo PCY, Lau SKP, Tang BSF, et al. Clinical evaluation of the polymerase chain reaction for the rapid diagnosis of tuberculosis. J Clin Pathol. 2004;57:281-5.

4. Bhat VS, Bhandary SK, Shenoy MS, Sathish Chandra BK, Girish BS. A Rare Case of Lupus Carcinoma of External Nose. Indian J Surg Oncol. 2011;2:215-7.

5. Arya S, Kushwaha R, Bunkar M, Jain S. Extensive lupus vulgaris: A variant of extrapulmonary tuberculosis. Indian J Paediatr Dermatology. 2015;17:161.

6. Singal A, Sonthalia S. Cutaneous tuberculosis in children: The Indian perspective. Indian J Dermatology, Venereol Leprol. 2010;76:494.

7. Afsar FS, Ozcelik S, Uysal SS, Ermete M, Afsar I. Primary inoculation tuberculosis: A report of a rare entity. Rev Soc Bras Med Trop. 2015;48:112-4.

8. Jesus G. Vallejo, Lydia T. Ong JRS. Clinical Features, Diagnosis, and Treatment of Tuberculosis in Infants. Pediatrics. 1994;94:1-7. 